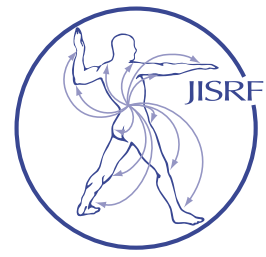

\title{
Is Utilizing a Modular Stemmed Tibial Component in Obese Patients Undergoing Primary Total Knee Replacement Cost-Effective?
}

Martin $J R^{1}$, Otero $J E^{2}$, Beaver $W B^{1}$, Springer $B D^{1}$, Griffin $W L^{1}$

\section{Abstract}

Background: There has been recent enthusiasm for the use of modular stemmed tibial components in obese (BMI $\geq 35 \mathrm{~kg} / \mathrm{m} 2$ ) patients undergoing primary total knee arthroplasty (TKA). This has been mainly driven by studies demonstrating statistically significant increases in the rates of aseptic tibial loosening (ATL) in this patient population. However, to our knowledge, no study has specifically evaluated the cost effectiveness of this current recommendation.

Methods: The following study was performed utilizing previously obtained data on the incidence of ATL in obese patients undergoing primary TKA. This data was then utilized to create a cost calculator that can evaluate the price point at which the use of a stemmed tibial component in all obese patients would be less than or equal to the costs of revision surgery if a stemmed implant was not utilized.

Results: Utilizing historical data with a revision rate of $4 \%$ for aseptic loosening of the tibia on obese patients, a cost calculator was developed. The cost calculator requires the input of expected or known incidence of ATL utilizing a stem extension and the expected or known costs of revision for ATL.

Conclusion: The following cost calculator quickly determines a price point at which the use of a tibial stem offsets the costs of revision surgery. While this study may not provide an exact cost-effectiveness of modular stem fixation due to model limitations, it will hopefully initiate the discussion for providing more cost-effective individualized care for this patient population.

\section{Background}

Obesity is currently an epidemic, affecting approximately one third of adults in the United States [1]. The average body mass index (BMI) in patients undergoing primary total knee arthroplasty (TKA) continues to increase. Numerous studies have demonstrated significantly worse outcomes in obese patients undergoing primary TKA [25]. More recently, several studies have begun to identify obesity as a risk factor for aseptic loosening of the tibial component after primary TKA [ $\underline{6}-\underline{8}]$. Abdel noted an approximately doubled risk of aseptic tibial loosening (ATL) in this patient population at 15 year follow-up [7]. They noted that increased implant fixation with the use of a tibial stem may decrease the rate of ATL in patients with a BMI $\geq 35 \mathrm{~kg} / \mathrm{m} 2$.

The introduction of a modular tibial stem significantly increases fixation of the tibial component. One study noted that the addition of a short tibial stem decreases proximal tibial cement-bone interface compressive stresses by

Keywords: obesity; total knee arthroplasty; cost calculator; stemmed tibial component; cost-effectiveness

Level of Evidence: AAOS Therapeutic Level IV 
$136 \%$ and cement-bone shear stress by $92 \%$ [9]. However, the use of a tibial stem has not been demonstrated to significantly improve outcomes in obese patients undergoing TKA [10]. Additionally, the incidence of ATL in obese patients that receive a modular tibial stem is unknown. Despite limited clinical evidence, there has been increased use of tibial stems in obese patients undergoing primary TKA to decrease the incidence of ATL.

Utilization of a tibial stem has several limitations including increased surgical time, increased difficulty of revision of the implant, and cost. The addition of a tibial stem is not available with all tibial baseplates. Some implants may require conversion from the standard primary tibial tray to a revision tibial tray. Conversion to a revision tibial tray may substantially change implant costs based on the cost differential of the primary and revision implant pricing. Currently, there is almost no data available if utilizing a modular stemmed tibial component for obese patients undergoing primary TKA is cost-effective. Therefore, the following study was designed to develop a cost-calculator that would evaluate the price point at which the use of a tibial stem would offset the cost of revision surgery in obese patients (BMI $\geq 35 \mathrm{~kg} / \mathrm{m} 2$ ) undergoing primary TKA. The cost calculator was then applied to a high volume arthroplasty center to determine the projected costs at this institution. While the following cost calculator only represents a very basic model of cost-effectiveness, the primary goal for this study is to begin the discussion in providing cost-effective personalized medicine for obese patients undergoing primary TKA.

\section{Materials and Methods}

The following study was performed utilizing historical data on the incidence of ATL in patients undergoing primary TKA [7]. This dataset included retrospectively collected information from 1998 to 2012 utilizing a variety of contemporary total knee arthroplasty designs. Four major implant manufacturers were included, specifically DePuy (Warsaw, IN), Stryker (Mahwah, NJ), Zimmer (Warsaw, IN), and Smith and Nephew (Memphis, TN). Stratifying aseptic loosening by BMI, obese patients had a statistically significant increased rate of aseptic loosening at 15 years $(4.26 \%$ vs. $2.16 \%)$ and a hazard ratio $2.3(\mathrm{p}=0.003)$. It should be noted that some primary implants have different implant geometry or stem lengths which may not have significantly higher rates of tibial loosening in obese patients, and this particular implant was not included in the study design 11.

The four percent incidence of ATL in obese patients at
15 years, with contemporary total knee arthroplasty designs, was then utilized as the main determinant for building the cost calculator. The following formula was utilized to determine if the costs of the addition of a tibial stem were cost effective:

$$
\mathrm{t}=\operatorname{Cos}^{\text {non-stemmed }}-\operatorname{Cos}^{\text {temmed }}
$$

$t$ represents the cost of the tibial stem. Cost $^{\text {non-stemmed }}$ represents the cost for revision surgery assuming the historical incidence of $4 \%$ for all obese patients undergoing primary TKA without a stemmed tibial component. Cost ${ }^{\text {stemmed }}$ represents the cost for revision surgery based on the incidence of revision when utilizing a stemmed tibial component in all patients.

For simplification of the scoring calculator, variables that could be easily identified at most institutions, including the incidence of ATL when a stemmed tibial component and the cost of revision for aseptic loosening were utilized. More sophisticated modeling would likely consider variables that are more challenging to quantify such as quality adjusted life years, pain scoring, vocational absences, costs of complications, etc. However, the aim of this study was only to determine at what price point the cost of the tibial stem offsets the cost of revision for ATL.

A scoring calculator was then developed that would calculate the cost of a tibial stem by allowing the user to insert two variables; 1 . the incidence of ATL when a modular tibial stem was utilized, and 2. the cost of revision surgery for ATL. An example was then utilized to demonstrate how the calculator might be applied. Additionally, the calculator was applied to a high volume joint center to determine if the costs of the modular tibial stem offset the costs of revision for aseptic loosening at each institution. It should be noted that at our institution, a short $(<40 \mathrm{~mm})$ supplemental cemented tibial stem was utilized for additional fixation. No long constructs or cementless designs were included.

\section{Results}

A scoring calculator was developed utilizing price points of revision surgery beginning at $\$ 5,000$ and increasing by $\$ 5,000$ intervals up to $\$ 50,000$. The rates of aseptic loosening were listed from $0 \%$ to $4.5 \%$. An aseptic loosening rate of $4 \%$ identified the point at which the use of a tibial stem could not add any additional cost to the total implant price or it would no longer be cost effective. Additionally, $4.5 \%$ was included only for completeness. It was not believed that the use of a tibial stem would result in a 
higher rate of aseptic loosening. For the creation of the table, the revision rate in patients that did not receive a stem was assumed to be $4 \%$. The following tibial stem cost calculator can be seen in Table 1.

\section{Case Example}

The cost calculator can be utilized to determine the cost-effectiveness of the stem when the incidence of aseptic loosening of the tibial implant is known as well as the cost of revision surgery for aseptic loosening of the tibia. Therefore, if a hospital has

Table 1: Cost Calculator Table

\begin{tabular}{|c|c|c|c|c|c|c|c|c|c|c|}
\hline & \multicolumn{7}{|l}{ Cost of Revision for aseptic loosening (dollars) } & & & \\
\hline $\begin{array}{c}\text { Aseptic } \\
\text { loosening } \\
\text { Incidence } \\
(\boldsymbol{\%})\end{array}$ & $\mathbf{5 0 0 0}$ & $\mathbf{1 0 0 0 0}$ & $\mathbf{1 5 0 0 0}$ & $\mathbf{2 0 0 0 0}$ & $\mathbf{2 5 0 0 0}$ & $\mathbf{3 0 0 0 0}$ & $\mathbf{3 5 0 0 0}$ & $\mathbf{4 0 0 0 0}$ & $\mathbf{4 5 0 0 0}$ & $\mathbf{5 0 0 0 0}$ \\
\hline $\mathbf{0}$ & 200 & 400 & 600 & 800 & 1000 & 1200 & 1400 & 1600 & 1800 & 2000 \\
\hline $\mathbf{1}$ & 150 & 300 & 450 & 600 & 750 & 900 & 1050 & 1200 & 1350 & 1500 \\
\hline $\mathbf{1 . 5}$ & 125 & 250 & 375 & 500 & 625 & 750 & 875 & 1000 & 1125 & 1250 \\
\hline $\mathbf{2}$ & 100 & 200 & 300 & 400 & 500 & 600 & 700 & 800 & 900 & 1000 \\
\hline $\mathbf{2 . 5}$ & 75 & 150 & 225 & 300 & 375 & 450 & 525 & 600 & 675 & 750 \\
\hline $\mathbf{3}$ & 50 & 100 & 150 & 200 & 250 & 300 & 350 & 400 & 450 & 500 \\
\hline $\mathbf{3 . 5}$ & 25 & 50 & 75 & 100 & 125 & 150 & 175 & 200 & 225 & 250 \\
\hline $\mathbf{4}$ & 0 & 0 & 0 & 0 & 0 & 0 & 0 & 0 & 0 & 0 \\
\hline $\mathbf{4 . 5}$ & -25 & -50 & -75 & -100 & -125 & -150 & -175 & -200 & -225 & -250 \\
\hline
\end{tabular}

a known incidence of $1.5 \%$ for ATL when a tibial stem is utilized and the costs for a revision for aseptic loosening are $\$ 30,000$ per revision; the tibial stem would need to be less than $\$ 750$. If the stems cost any more than $\$ 750$ the cost of revision for ATL would be less than the total costs for the use of stems.

$$
\begin{gathered}
\mathrm{t}=\operatorname{Cos}^{\text {non-stemmed }}-\text { Cost }^{\text {stemmed }} \\
\mathrm{t}=4 \%(\$ 30,000)-1.5 \%(\$ 30,000) \\
\mathrm{t}=\$ 1,200-\$ 450 \\
\mathrm{t}=\$ 750
\end{gathered}
$$

\section{High Volume Center}

The following institution has less than a $1 \%$ incidence of ATL when utilizing a tibial stem. Therefore, 1\% ATL was utilized for the following calculation. The cost of revision surgery for ATL is approximately $\$ 45,000 /$ revision (based on average hospital collections). Therefore, the cost calculator demonstrates that the cost of the tibial stem should be $\$ 1,350$. However, at this institution, the costs for the tibial stem are $\$ 2,000 /$ stem. Therefore, each additional case where a tibial stem is utilized represents an additional $\$ 650 /$ case in costs.

$$
\begin{gathered}
\mathrm{t}=4 \%(\$ 45,000)-1 \%(\$ 45,000) \\
\mathrm{t}=\$ 1,800-\$ 450 \\
\mathrm{t}=\$ 1,350
\end{gathered}
$$

\section{Discussion}

Obesity remains a substantial risk factor for complications and worse outcomes following primary TKA $[\underline{4}, \underline{12}-$ 15]. Many studies have associated obesity with increased implant loosening rates, but there remains a paucity of data regarding the economic considerations in this patient population $[\underline{6}, \underline{8}]$. A recent study by Abdel et. al. has recommended considering the use of a stemmed tibial component in obese patients undergoing primary TKA [7]. While this may potentially reduce the incidence of ATL in this patient population, we believe that this practice may significantly increase the cost of joint replacements if appropriate implant pricing has not been considered. Therefore, the following study provides a framework for implant pricing to begin the discussion of improving cost-effectiveness in obese patients undergoing primary TKA.

Recent studies have demonstrated that the average BMI in patients undergoing primary TKA has continued to rise [16]. The average BMI is approaching $35 \mathrm{~kg} / \mathrm{m} 2$. If a modular stemmed tibial component was utilized in all patients with a BMI $\geq 35 \mathrm{~kg} / \mathrm{m} 2$, approximately $50 \%$ of patients would require this implant. If cost-effectiveness is not considered, there is a potential for these implants to add excessive additional costs. This would be contradictory to the current emphasis that has been placed on cost-containment in total joint replacement. Therefore, this study critically analyzes the costs of utilizing a stemmed tibial component in obese patients undergoing primary TKA with a goal of providing more cost-effective individualized care.

The cost-effectiveness of the tibial stem in this model is dependent on multiple factors. First, the incidence of ATL is extremely important. The lower the incidence of ATL in the obese population implanted without stems, the greater the number needed to treat to prevent this complication and therefore increased costs. Next, the incidence of aseptic loosening in obese patients in which a tibial stem was utilized can dramatically alter the cost-effectiveness. In an ideal scenario, the use of a tibial stem would prevent all 
cases of tibial loosening. However, realistically, there will be a small percentage of patients that still develop ATL in this patient population. Preferably, this percentage would not be greater than the incidence of loosening in patients where a tibial stem was not utilized. One final consideration would be the cost of revision surgery for aseptic loosening. The more expensive the revision surgery, the more cost-effective the stem can potentially become.

Current pricing for tibial implants is commonly negotiated between hospital systems and the implant manufacturers. A thorough understanding of each implant's revision options is therefore necessary. Some implant models, can readily incorporate a stem on their primary tibial tray. However, other tibial implants are not modular and require conversion to a revision tibial tray. There may be substantial cost differences between adding a stem and changing to a revision tibial tray. Therefore, the modularity or ability to accept a tibial stem must be factored into the cost-effectiveness of supplemental tibial stem fixation. It should be noted that decreasing 90-day complication and readmission rates is one of the main focuses of many hospital systems. However, preventing aseptic loosening years later may be overlooked in many discussions on implant pricing but should be considered.

As was identified in the clinical example, the cost of a tibial stem was not cost-effective based on this simplified cost calculator. The high volume center had an increased cost of $\$ 650 /$ stem. It is possible that other variables, including quality of life, could have been modeled into the cost-analysis and provided more favorable results for the use of a modular tibial stem. However, there is currently limited clinical evidence that supports the benefits for the use of a tibial stem. Therefore, further reductions in the price of a tibial stem may be necessary to increase the costeffectiveness of these implants. Considering that the average BMI is now approaching $35 \mathrm{~kg} / \mathrm{m} 2$, approximately 300,000 of the estimated 600,000 annual TKAs will likely require a tibial stem. The use of tibial stem may increase the costs by approximately $\$ 200$ million utilizing this data. However, the main objective of this study is not necessarily to recommend for or against the use of modular tibial stems. The goal is to initiate the discussion of cost-effectiveness of global use of these stems in all obese patients, with a focus on individualized care.

Currently, there is a small margin for cost-effectiveness of the tibial stem based on this model. When implant costs rise above a certain threshold, the tibial stem can add substantial costs to the surgery. Unfortunately, as the average BMI in patients undergoing primary TKA increases, this will further increase the costs. Therefore, more selective utilization of tibial stems in obese patients may further im- prove cost-effectiveness of the implant. Fehring et. al. recently identified smaller implant size and obesity to be associated with aseptic tibial varus collapse []]. He noted that higher tibial stress appears to be a risk factor for this failure mechanism and recommended consideration of a stem for additional fixation at a critical tibial stress threshold of 300,000 Pa. Subsequently, Martin et. al. noted that preoperative varus deformity may also be associated with aseptic tibial varus collapse []]. Screening patients for risk factors associated with ATL (smaller implant size and preoperative varus deformity) will improve the cost-effectiveness of the tibial stem.

While this study represents the first attempt to demonstrate the cost-effectiveness of utilizing a tibial stem in obese patients undergoing primary TKA, there are several limitations. First, this study utilizes a basic model that attempts to determine the cost-effectiveness of utilizing a stemmed tibial component. The modeling process has inherent limitations and is not as accurate as a randomized prospective trial. However, the time and cost-savings of the model are notable and does spark the debate of the cost-effectiveness of modular stem fixation in obese patients undergoing primary TKA. Unfortunately, this model does not address all of the variables but may lead to improved modeling in the future. Second, implant pricing is not universal. Higher volume centers will likely be able to obtain implants at a reduced cost. This can impact the cost-effectiveness models by increasing the price of the implants as well as the cost of revision. Third, the rate of ATL is based on historic controls. Higher rates of aseptic loosening will increase the cost-effectiveness of the tibial stem. Additionally, the ability of the modular tibial stem to prevent ATL is not known. As the rate of revision for aseptic loosening of the stemmed tibial component approaches the rate of aseptic loosening without a stem, the additional costs of the stem will eventually become cost-ineffective at any price. Finally, this study attempts to simplify the complexities of calculating cost-effectiveness. It is very challenging to determine an exact cost associated with revision surgery. The costs represented in this study do not factor in patient's debility before or after surgery, secondary effects of caregivers, the need for nursing/rehab facility use, or post-operative complications that are not captured in the 90-day global period, or the costs of work absences $[17, \underline{18}]$. As these other variables are included, the cost-effectiveness of the modular tibial stem will likely increase as well. The authors acknowledge this as a substantial limitation, and understand that further investigation is necessary on this topic to build more accurate models. However, with the recent push for cost-containment in arthroplasty surgeries, there will be greater scrutiny on the costs of im- 
plants. Therefore, this study offers an introductory view into the cost-effectiveness of tibial stems.

\section{Conclusion}

Utilization of a tibial stem in obese patients has been advocated as a method for decreasing the rates of aseptic loosening in primary TKA. Currently, there are few clinical studies that have demonstrated a significant reduction in aseptic loosening with a modular stemmed tibial component. However, theoretical benefits have led many surgeons to adopt this methodology. The following cost calculator was proposed as a method for rudimentarily calculating the cost-effectiveness of utilizing a tibial stem. This study is not meant to specifically determine implant pricing, but rather to re-evaluate clinical practices in order to provide more cost-effective individualized medicine. We therefore would recommend more selective utilization of tibial stems in obese patients undergoing primary TKA whenever possible to improve the cost-effectiveness of these implants.

\section{References}

1. Campos P, Saguy A, Ernsberger P, Oliver E, Gaesser G. The epidemiology of overweight and obesity: public health crisis or moral panic? Int J Epidemiol. 2006;35(1):55-60. doi:10.1093/ije/dyi254.

2. Martin JR, Watts CD, Taunton MJ. Bariatric surgery does not improve outcomes in patients undergoing primary total knee arthroplasty. Bone Joint J. 2015;97B(11):1501-1505. doi:10.1302/0301-620X.97B11.36477.

3. Wagner, E.R.; Kamath A.F., Fruth, K; Harmsen WS. BDJ. Influence of Body Mass Index on Total Knee Arthroplasty Outcomes. J Bone Jt Surg. 2015.

4. D'Apuzzo MR, Novicoff WM, Browne JA. The John Insall Award: Morbid obesity independently impacts complications, mortality, and resource use after TKA. Clin Orthop Relat Res. 2015;473(1):57-63. doi:10.1007/s11999-014-3668-9.

5. Mason JB, Callaghan JJ, Hozack WJ, Krebs V, Mont MA, Parvizi J. Obesity in Total Joint Arthroplasty: An Issue With Gravity. J Arthroplasty. 2014;29(10):1879. doi:10.1016/j.arth.2014.09.002.

6. Martin JR, Fehring KA, Watts CD, Springer BD, Fehring TK. Radiographic Findings in Patients With Catastrophic Varus Collapse After Total Knee Arthroplasty. $J$ Arthroplasty. 2018;33(1):241-244. doi:10.1016/j.arth.2017.08.014.

7. Abdel MP, Bonadurer GF, Jennings MT, Hanssen AD. Increased Aseptic Tibial Failures in Patients With a BMI $\geq 35$ and Well-Aligned Total Knee Arthroplasties. J Arthroplasty. July 2015. doi:10.1016/j.arth.2015.06.057.

8. Fehring TK, Fehring KA, Anderson LA, Otero JE, Springer BD. Catastrophic Varus Collapse of the Tibia in Obese Total Knee Arthroplasty. J Arthroplasty. 2017;32(5):1625-1629. doi:10.1016/j.arth.2016.12.001.

9. Gopalakrishnan A, Hedley AK, Kester MA. Magnitude of cement-device interfacial stresses with and without tibial stemming: impact of BMI. J Knee Surg. 2011;24(1):3-8. http://www.ncbi.nlm.nih.gov/pubmed/21618932. Accessed July $10,2017$.

10. Parratte S, Ollivier M, Lunebourg A, Verdier N, Argenson JN. Do Stemmed Tibial Components in Total Knee Arthroplasty Improve Outcomes in Patients With Obesity? Clin Orthop Relat Res. 2017;475(1):137-145. doi:10.1007/s11999-0164791-6.

11. Crawford DA, Berend KR, Nam D, Barrack RL, Adams JB, Lombardi Jr A V. Low Rates of Aseptic Tibial Loosening in Obese Patients With Use of High-Viscosity Cement and Standard Tibial Tray: 2-Year Minimum Follow-Up. 2017. doi:10.1016/j.arth.2017.04.018.

12. Abdel MP, Ast MP, Lee Y-Y, Lyman S, González Della Valle A. All-cause in-hospital complications and urinary tract infections increased in obese patients undergoing total knee arthroplasty. J Arthroplasty. 2014;29(7):1430-1434. doi:10.1016/j. arth.2014.02.013.

13. Alvi HM, Mednick RE, Krishnan V, Kwasny MJ, Beal MD, Manning DW. The Effect of BMI on 30 Day Outcomes Following Total Joint Arthroplasty. J Arthroplasty. 2015;30(7):1113-1117. doi:10.1016/j.arth.2015.01.049.

14. Dowsey MM, Choong PFM. Obese diabetic patients are at substantial risk for deep infection after primary TKA. Clin Orthop Relat Res. 2009;467(6):15771581. doi:10.1007/s11999-008-0551-6.

15. Thelwall S, Harrington P, Sheridan E, Lamagni T. Impact of obesity on the risk of wound infection following surgery: results from a nationwide prospective multicentre cohort study in England. Clin Microbiol Infect. July 2015. doi:10.1016/j. cmi.2015.07.003.

16. Odum SM, Springer BD, Dennos AC, Fehring TK. National obesity trends in total knee arthroplasty. J Arthroplasty. 2013;28(8 Suppl):148-151. doi:10.1016/j. arth.2013.02.036.

17. Maradit Kremers H, Visscher SL, Moriarty JP, et al. Determinants of Direct Medical Costs in Primary and Revision Total Knee Arthroplasty. Clin Orthop Relat Res. 2013;471(1):206-214. doi:10.1007/s11999-012-2508-z.

18. Nichols CI, Vose JG. Clinical Outcomes and Costs Within 90 Days of Primary or Revision Total Joint Arthroplasty. J Arthroplasty. 2016;31(7):1400-1406.e3. doi:10.1016/j.arth.2016.01.022.

SUBMISSION HISTORY

Submitted October 24, 2018

Reviewed November 15, 2018

Revised December 11, 2018

Accepted December 17, 2018

Published December 31, 2018

AUTHOR AFFILIATIONS

1 J. Ryan Martin, MD; Walter Beaver, MD; Bryan Springer, MD ; William Griffin, MD OrthoCarolina, Hip and Knee Center, 2001 Vail Avenue Suite 200A, Charlotte, NC 28207

2 Jesse Otero, MD, PhD University of lowa Carver College of Medicine, 200 Hawkins Drive, lowa City, IA 52242-1009

(Direct inquires to Ryan Martin, johrmart@gmail.com)

\section{AUTHOR DISCLOSURES}

The authors declare that there are no disclosures regarding the publication of this paper.

\section{COPYRIGHT \& OPEN ACCESS}

(c) 2018 Martin, Otero, Beaver, Springer, Griffin. All rights reserved. Authors retain copyright and grant the journal right of first publication with the work. Reconstructive Review is an open access publication and follows the Creative Commons Attribution-NonCommercial CC BY-NC. This license allows anyone to download works, build upon the material, share them with others for non-commercial purposes as long as they credit the senior author, Reconstructive Review, and the Joint Implant Surgery \& Research Foundation (JISRF). An example credit would be: "Courtesy of (senior author's name), Reconstructive Review, JISRF, Chagrin Falls, Ohio". 